\title{
Thrombus on the Tricuspid Valve in a Patient With Primary Antiphospholipid Syndrome After Implantation of an Inferior Vena Cave Filter
}

\author{
Mio Ebato, MD; Hitomi Kitai, BE*; Hideko Kumakura, BE*; \\ Yoshiko Nakamura, MD; Nobuyuki Shimizu, MD**; \\ Youichi Takeyama, MD**
}

\begin{abstract}
A 62-year-old woman with a history of pulmonary embolism and primary antiphospholipid syndrome (PAPS) with positivity for lupus anticoagulant was admitted to hospital with shortness of breath. A filter had been implanted in her inferior vena cava (IVC) 5 years previously. Emergency echocardiography revealed a lobulated, mobile echogenic mass on the tricuspid valve, and on pulmonary perfusion scintigraphy several apparently new defects were noted. Fibrinolytic therapy improved her symptoms and the pulmonary perfusion, then intravenous heparinization was continued for a further week. Repeat echocardiography performed on the 7th day of the admission showed complete disappearance of the mass, which was retrospectively diagnosed as a thrombus based on its resolution with fibrinolytic and anticoagulant therapies. (Circ J 2002; 66: 425-427)
\end{abstract}

Key Words: Inferior vena cava; Primary antiphospholipid syndrome; Thrombus; Tricuspid valve

A ntiphospholipid syndrome (APS) is a combination of the clinical symptoms of arterial and venous thromboembolism and the presence of autoantibodies such as anticardiolipin $\beta 2$ GPI antibody, the lupus anticoagulant and the antibodies responsible for the falsepositive serologic test result for syphilis. Primary APS (PAPS) is defined by the presence of these antibodies and thromboembolic phenomena without systemic lupus erythematosus (SLE) and other disease processes. Intracardiac thrombi have been reported ${ }^{1-7}$ but involvement of the tricuspid valve is extremely rare 5 We report a case of PAPS with a lobulated thrombus on the tricuspid valve, which formed after implantation of a filter in the inferior vena cava (IVC) and resolved with fibrinolytic therapy.

\section{Case Report}

A 62-year-old woman was admitted with progressive shortness of breath and tachypnea. The patient's medical history included a 15-year history of bronchial asthma, and a pulmonary embolism caused by a deep vein thrombus 5 years previously. At that time, computed tomography (CT) revealed a large thrombus in the main pulmonary artery, which dissolved with intravenous administration of tissue plasminogen activator. Although several perfusion-ventilation mismatch areas remained on the pulmonary perfusion scan after the therapy (segment 6 on the left, segment 8 on the right), she was discharged with no symptoms after the implantation of an IVC filter. Further evaluation during

(Received February 2, 2001; revised manuscript received June 1, 2001; accepted June 25, 2001)

Division of Clinicopathology, *Department of Central Clinical Laboratory and **Division of Cardiology, Showa University Fujigaoka Hospital, Yokohama, Japan

Mailing address: Mio Ebato, MD, Division of Clinicopathology, Showa University Fujigaoka Hospital, 1-30 Fujigaoka, Aoba-ku, Yokohama 227-8501, Japan that admission led to the diagnosis of PAPS with positivity for lupus anticoagulant. The anticardiolipin $\beta 2$ GPI antibody level was less than $1.2 \mathrm{U} / \mathrm{ml}$. Antinuclear antibodies were negative, and the activities of proteins $\mathrm{C}$ and $\mathrm{S}$ were normal, consistent with the diagnosis. She had no history of spontaneous abortion and had delivered normally with 3 pregnancies. After discharge, she was treated with warfarin with a target international normalized ratio (INR) of 2.0 at an outpatient clinic.

One year later, she was admitted to examine her pulmonary pressure and right heart pressure, which were found to be in the normal range. A repeat pulmonary perfusion scan showed no change. She continued to be positive for lupus anticoagulant at the second admission. Since then, she has been followed at an outpatient clinic and managed with warfarin bronchodilator and warfarin. However, the warfarin therapy was discontinued 10 months prior to her third admission.

On the third admission, she had an oxygen saturation of $89 \%$, a respiratory rate of 28 breaths/min, and a 12 -lead ECG that showed an inverted $T$ wave in leads $V_{1-3}$. Chest $\mathrm{X}$-ray suggested dilatation of the right pulmonary artery. Laboratory tests revealed a white blood cell count of $12,500 / \mathrm{mm}^{3}$, hematocrit $41.7 \%$, and platelet counts 124,000 $/ \mathrm{mm}^{3}$. Transthoracic echocardiography revealed a $1.7 \times 1.8$ $\mathrm{cm}$, lobulated, isoechoic, highly mobile mass on the septal leaflet of the tricuspid valve (Fig 1). The right atrium and ventricle were dilated and mild tricuspid regurgitation was detected, with an estimated pulmonary artery systolic pressure of $65 \mathrm{mmHg}$. Her left heart valves were normal and there were no abnormalities in the left ventricular wall motion. Pulmonary perfusion scan revealed a new perfusion defect in the left upper lobe and global hypoperfusion of the right lobe in addition to the previously detected defects (Fig 2).

Myxoma and nonbacterial vegetation were considered in the differential diagnosis of the mass and surgical removal 
A

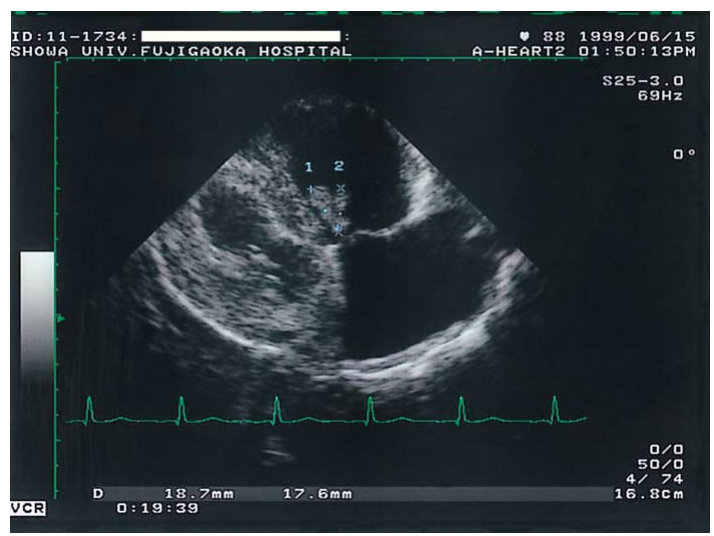

B

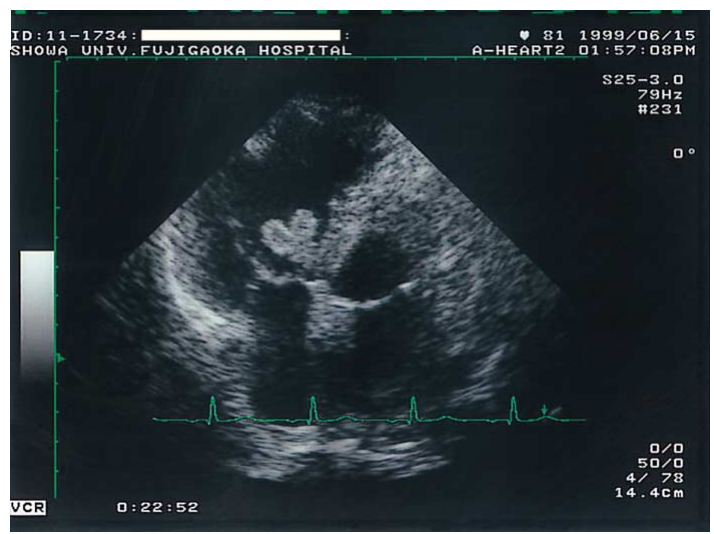

Fig 1. (A,B) Echocardiography. An isoechoic, lobulated, mobile mass can be seen on the edge of the septal leaflet of the tricuspid valve.

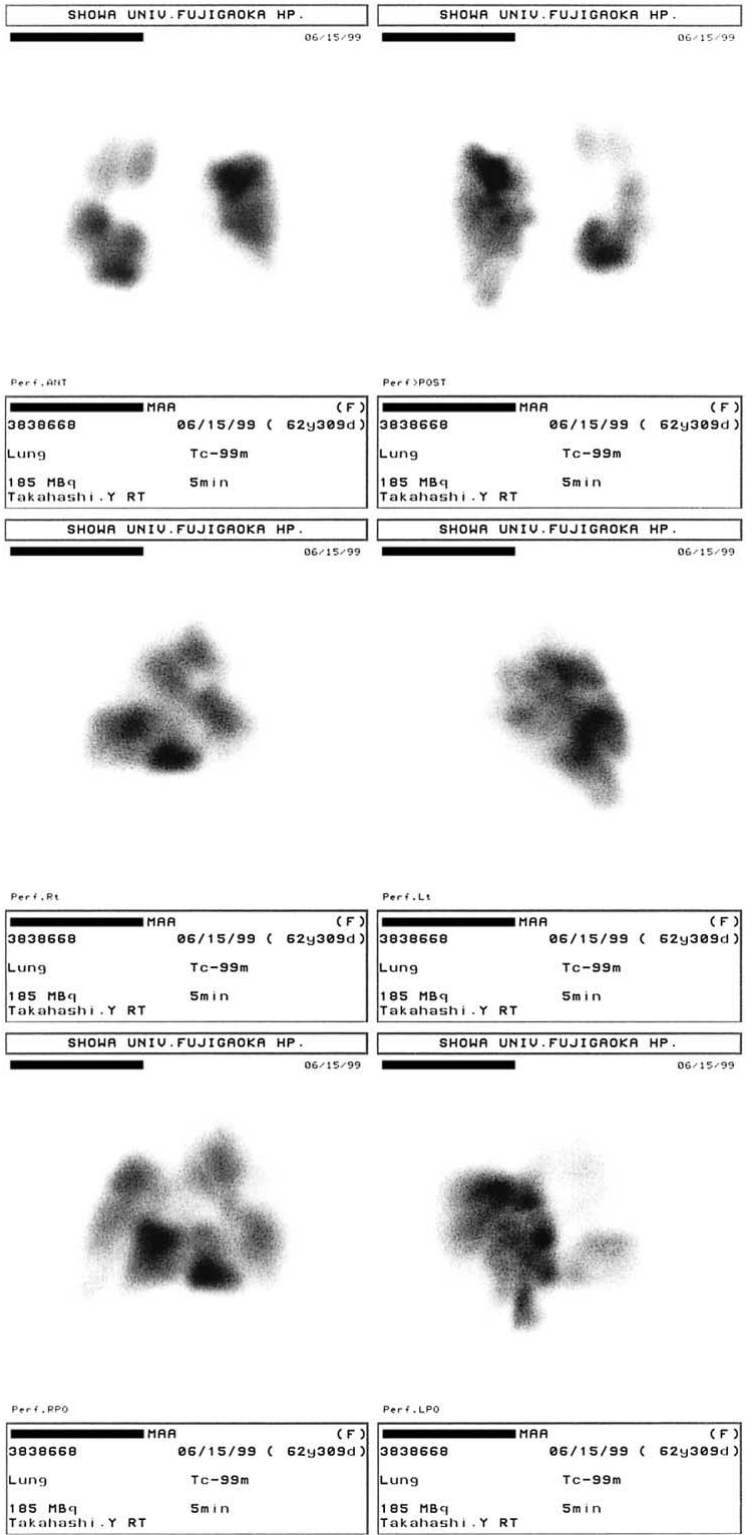

Fig 2. Pulmonary perfusion scans done during the present admission. In addition to the partial subsegmental hypoperfusion in segment 6 on the left, and perfusion defects in segment 8 on the right, which had been observed previously, new defects in the left upper lobe and hypoperfusion in segment 1 in the right upper lobe were found.

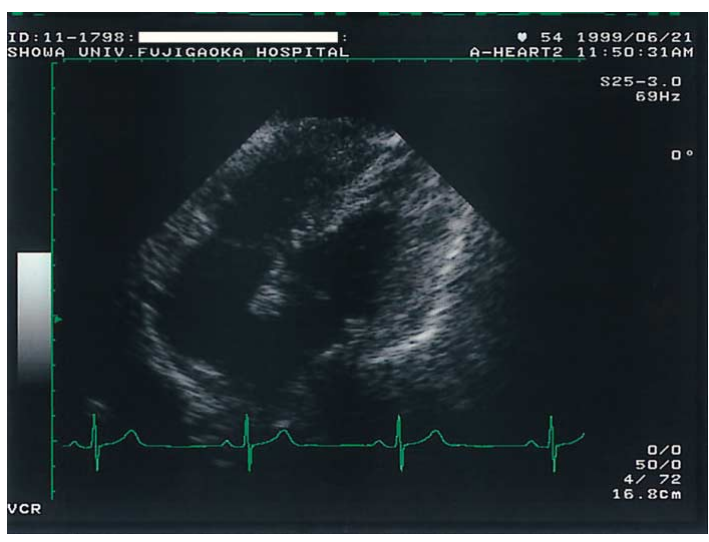

Fig 3. Echocardiography on the seventh day of the current admission. The mass on the tricuspid valve had completely disappeared after fibrinolytic and anticoagulant therapy. Tricuspid regurgitation had become trivial and its speed had normalized.

of the mass was discussed. However, because of her rapidly worsening symptoms and their similarity to those of her previous admission, it was felt that immediate medical therapy was necessary. Tissue plasminogen activator $(1,800,000 \mathrm{IU}$ of Alteplase) was given intravenously and soon after, her symptoms began to resolve and her blood gas levels improved.

On the second day of admission, the oxygen saturation, which had improved to $97 \%$ after fibrinolytic therapy under oxgenation, decreased to $92 \%$ although she had no symptoms of hypoxemia. A repeat pulmonary perfusion scan showed new defects in the right lower lobe with improvement in the perfusion of the left upper lobe. Alteplase $(1,200,000 \mathrm{IU})$ was again infused followed by intravenous heparinization. Later that day, echocardiography was performed and the mobile mass appeared to be smaller in size. Heparin infusion was continued for 6 days followed by warfarin as anticoagulant therapy.

On the seventh hospital day, repeat echocardiography demonstrated complete dissolution of the mass on the tricuspid valve (Fig 3) and the tricuspid regurgitation had also decreased to a trivial level with a peak speed normalized to $2.4 \mathrm{~m} / \mathrm{s}$. Pulmonary perfusion scan showed further improvements in the left upper and right lower lobes. Right heart catheterization performed 3 weeks after the admission demonstrated normal pulmonary artery pressure, right ventricular pressure and right atrial pressure. Fourteen 
months later, she remains free of symptoms without any thromboembolic episodes while continuing warfarin with a target INR 2.0-2.5.

\section{Discussion}

The prevalence of valvular disease in PAPS has been reported to be between 35\% and 75\% - $^{-11}$ The echocardiographic findings include valvular thickening and nonbacterial vegetation on the left heart valves- ${ }^{8-16}$ Tricuspid valve lesions have been reported in a few patients with APS. Sastry reported isolated tricuspid valve vegetation with severe tricuspid regurgitation in a patient with APS secondary to SLE and histopathologic examination of the excised valve showed diffuse, thickened leaflets with commissure fusion! ${ }^{17}$

The efficacy of anticoagulant therapy on vegetative lesions is controversial;11-16 some authors have suggested that vascular proliferation, infiltration of fibroblasts, necrosis and calcification are the predominate factors involved in the etiopathogenesis of echogenic masses on the left heart valves in patients with APS 11,15 and would therefore not be expected to respond to fibrinolytic and anticoagulant therapies, whereas others have reported success in treating these vegetations with anticoagulant therapy!2-14

In addition to the studies of valvular lesions, mural endocardial thrombi have been documented in patients with APS, ${ }^{1-7}$ in some cases, on normal-functioning right and left ventricles, 4 There have been few reports of thrombus on the tricuspid valve in patients with APS; Day et al reported a right atrial thrombi associated with PAPS, one of which was adherent to the edge of the anterior leaflet of the tricuspid valve?

In the present patient's case, the mass shown by echocardiography was considered to be a thrombus because it dissolved upon fibrinolytic and anticoagulant therapy. Right heart entrapment of pulmonary emboli in-transit has been reported ${ }^{18}$ but considering the size of the thrombus in the presence of an IVC filter placed during a previous admission, the current thrombus may have formed in the right atrium and moved to the tricuspid valve or it may have formed directly on the ventricular side of the tricuspid valve.

In patients with APS, careful use of anticoagulant therapy is necessary to prevent life-threatening systemic complications and pulmonary embolization. There is a high risk of recurrence after discontinuation of oral anticoagulant therapy, ${ }^{19-23}$ and in the present patient pulmonary embolism recurred 10 months after cessation of warfarin. Therefore, long-term anticoagulant therapy is thought to be necessary. The appropriate dose of warfarin in such patients is controversial. Lifelong treatment with oral anticoagulants with a target INR of 3.0 or more has been recommended, based on data from 3 retrospective studies, ${ }^{19}$ but several recent reports have suggested that many patients with APS can be treated with conventional doses of warfarin ${ }^{21-23,25}$ The risk of high-intensity anticoagulant therapy in patients with APS has also been reported ${ }^{26}$ The present patient's course has been uneventful for 14 months on a target INR of 2.0-2.5 after the most recent episode. Large-scale prospective studies will identify the optimal treatment for individual patients with APS.

\section{References}

1. Lubbe WF, Asherson RA. Intracardiac hrombus in systemic lupus erythematosus associated with lupus anticoagulant. Arthritis Rheum 1988; 31: 1453-1454.

2. Coppock MA, Safford RE, Danielson GK. Intracardiac thrombosis, phospholipid antibodies, and two-chambered right ventricule. $\mathrm{Br}$ Heart J 1988; 60: 455-458.

3. Leventhal LJ, Borofsky MA, Bergey PD, Schumacher HR. Antiphospholipid antibody syndrome with right atrial thrombosis mimicking an atrial myxoma. Am J Med 1989; 87: 111-113.

4. O'Hickey S, Skinner C, Beattie J. Life-threatening right ventricular thrombosis in association with phospholipid antibodies. Br Heart J 1993; 70: 279-281.

5. Day SM, Rosenzweig BP, Kronzon I. Transesophageal echocardiographic diagnosis of right atrial thrombi associated with the antiphospholipid syndrome. J Am Soc Echocardiogr 1995; 8: 937-940.

6. Bruce D. Left ventricular thrombi in a patient with the antiphospholipid syndrome. Br Heart J 1995; 74: 202-203.

7. Plein D, Camp GV, Efira A, Brunet A, Vandenbossche J. Intracardiac thrombi associated with antiphospholipid antibodies. J Am Soc Echocardiogr 1996; 9: 891-893.

8. Brenner B, Blumenfeld Z, Markiewicz W, Reisner SA. Cardiac involvement in patients with primary antiphospholipid syndrome. $J$ Am Coll Cardiol 1991; 18: 931-936.

9. Kaplan SD, Chartash EK, Pizzalello RA, Furie RA. Cardiac manifestations of antiphospholipid syndrome. Am Heart J 1992; 124: 1331 1338.

10. Garcia-Torres R, Amigo MC, De la Rosa A, Maron A, Reyes PA. Valvular heart disease in primary antiphospholipid syndrome (PAPS): Clinical and morphological findings. Lupus 1996; 5: 56-61.

11. Galve E, Ordi J, Barquinwro J, Evangelsta A, Vilardell M, Soler JS. Valvular heart disease in the primary antiphospholipid syndrome. Ann Intern Med 1992; 116: 293-298.

12. O'Neill D, Magaldi J, Dobkins D, Greco T. Dissolution of intracardiac mass lesions in the primary antiphospholipid antibody syndrome. Arch Intern Med 1995; 155: 325-327.

13. Skyme-Jones ARP, Wardrop CAJ, Wiles CM, Fraser AG. Transesophageal echocardiographic demonstration of resolution of mitral vegetation after warfarin in a patient with primary antiphospholipid syndrome. J Am Soc Echocardiogr 1995; 8: 251-256.

14. Agirbasli MA, Hansen DE, Byrd BFÖ. Resolution of vegetations with anticoagulation after myocardial infarction in primary antiphospholipid syndrome. J Am Soc Echocardiogr 1997; 10: 877-880.

15. Zavaleta NE, Barron JV, Galvis TC, Cruz FC, Cardenas AR, Keirns $\mathrm{C}$, et al. Echocardiographic evaluation of patients with primary antiphospholipid syndrome. Am Heart J 1999; 137: 974-979.

16. Grondin F, Giannoccaro JP. Antiphospholipid antibody syndrome associated with large aortic valve vegetation and stroke. Can J Cardiol 1995; 11: 133-135.

17. Sastry BK. Isolated tricuspid valve disease in a patient with elevated levels of anticardiolipin antibodies. Indian Heart J 1997; 49: 87-89.

18. Farfel Z, Shecter M, Vered Z, Rath S, Goor D, Gafni J. Review of echocardiographically diagnosed right heart entrapment of pulmonary emboli-in-transit with emphasis on management. Am Heart J 1987; 113: $171-178$

19. Khamashta MA, Cuadrado MJ, Mujic F, Taub NA, Hunt BJ, Hughes GR. The management of thrombosis in the antiphospholipid-antibody syndrome. N Engl J Med 1995; 332: 993-997.

20. Derksen RH, de Groot PG, Kater L, Nieuwenhuis HK. Patients with antiphospholipid antibodies and venous thrombosis should receive long term anticoagulant treatment. Ann Rheum Dis 1993; 52: 689692.

21. Rance A, Emmerich J, Fiessinger JN. Anticardiolipin antibodies and recurrent thromboembolism. Thromb Haemost 1997; 77: 221-222.

22. Prandoni P, Simioni P, Girolami A. Antiphospholipid antibodies, recurrent thromboembolism, and intensity of warfarin anticoagulation. Thromb Haemost 1996; 75: 859.

23. Shulman S, Sevenungsson E, Granqvist S. Anticardiolipin antibodies predict early recurrence of thromboembolism and death among patients with venous thromboembolism following anticoagulant therapy: Duration of Anticoagulation Study Group. Am J Med 1998; 104: $332-338$.

24. Rosove MH, Brewer PM. Antiphospholipid thrombosis: Clinical course after the first thrombotic event in 70 patients. Ann Intern Med 1992; 117: 303-308.

25. Derksen RHWM, de Groot PG. Do we know which patients with the antiphospholipid syndrome should receive long term high dose anticoagulation? J Autoimmun 2000; 15: 261-264.

26. Al Sayegh FA, Ensworth S, Huang S, Stein HB, Kinkhoff AV. Hemorrhagic complications of long-term anticoagulant therapy in seven patients with systemic lupus erythematosus and antiphospholipid syndrome. J Rheumatol 1997; 24: 1716-1718. 